\title{
Barriers and facilitators to exercise among adult cancer survivors in Singapore
}

\author{
Alexandre Chan ${ }^{1,2}$ (D) $\cdot$ Kayleen Ports ${ }^{1} \cdot$ Patricia Neo $^{3} \cdot$ Mothi Babu Ramalingam ${ }^{4} \cdot$ Ang Tee Lim $^{5} \cdot$ Benedict Tan $^{5}$. \\ Nicolas H. Hart ${ }^{6,7,8,9} \cdot$ Raymond J. Chan ${ }^{6,7} \cdot$ Kiley Loh $^{10}$
}

Received: 6 October 2021 / Accepted: 31 January 2022 / Published online: 14 February 2022

(c) The Author(s) 2022

\begin{abstract}
Purpose Exercise can help cancer survivors manage sequela, treatment side effects, improve overall quality of life, and is recommended for most. The purpose of this study was to investigate exercise behavior and factors influencing exercise engagement among cancer survivors at the National Cancer Centre, Singapore (NCCS).

Methods This cross-sectional study was inclusive of survivors of all cancer types and stages who were at least 21 years of age and had undergone chemotherapy at the NCCS. Surveys were utilized to assess survivor barriers and facilitators to exercise and to retrospectively assess physical activity and exercise behaviors at 4 cancer-related time periods (pre-diagnosis and post-diagnosis before, during, or after chemotherapy).

Results A total of 102 cancer survivors were enrolled; $60 \%$ were diagnosed with stage IV cancer. Predominant cancer types included lower gastrointestinal tract (25.5\%) and breast cancer (21.6\%). Prior to cancer diagnosis, $90.2 \%$ of participants reported aerobic activity satisfying NCCN guidelines. Significant reductions in reported exercise, and physical activity, were observed following cancer diagnosis that persisted during chemotherapy. Key exercise facilitators included the desire to remain healthy $(86.3 \%)$ and to improve sleep and mental well-being $(73.5 \%)$. Key barriers included side effects of treatment (52.0\%). Only $46.1 \%$ of survivors reported receiving exercise guidance from healthcare professionals following diagnosis. Conclusion Overall, even among this notably active cohort of Singaporean survivors, opportunities for increased exercise engagement throughout the survivorship continuum remain. Increased education regarding the benefits of exercise to survivors as well as guidance regarding exercise modalities including resistance training is greatly needed as well.
\end{abstract}

Keywords Oncology $\cdot$ Exercise $\cdot$ Cancer survivorship $\cdot$ Counseling $\cdot$ Barriers $\cdot$ Facilitators

Alexandre Chan

a.chan@uci.edu

1 Department of Clinical Pharmacy Practice, School of Pharmacy \& Pharmaceutical Sciences, University of California, Irvine, USA

2 Department of Oncology Pharmacy, National Cancer Centre Singapore, Singapore, Singapore

3 Division of Supportive and Palliative Care, National Cancer Centre Singapore, Singapore, Singapore

4 Department of Rehabilitation Medicine, Singapore, Singapore

5 Department of Sport and Exercise Medicine, Changi General Hospital, Singapore, Singapore
6 Caring Futures Institute, College of Nursing and Health Sciences, Flinders University, Adelaide, South Australia, Australia

7 Cancer and Palliative Care Outcomes Centre, Queensland University of Technology, Brisbane, QLD, Australia

8 Exercise Medicine Research Institute, Edith Cowan University, Joondalup, WA, Australia

9 Institute for Health Research, University of Notre Dame Australia, Fremantle, WA, Australia

10 Division of Medical Oncology, National Cancer Centre Singapore, Singapore, Singapore 


\section{Introduction}

Advancements in cancer detection and treatment have led to decreased cancer mortality rates and a rapidly increasing population of cancer survivors with unique survivorship needs [1]. Cancer survivors, defined by the National Comprehensive Cancer Network (NCCN) as individuals from cancer diagnosis through end of life, often experience reduced quality of life (QoL) due the physiological and psychosocial side effects associated with cancer and its treatments. Prevalent sequelae and side effects include cancer-related fatigue, pain, depression, cognitive difficulties, and sleep disruption that often persist years after treatment completion, in addition to treatment-related comorbidities including cardiovascular disease and type II diabetes [2-4]. The prevalence of serious and often long-term morbidities among survivors, especially those who have undergone chemotherapy, highlights the critical need for effective interventions. One promising intervention of growing interest is exercise.

A growing body of evidence has determined exercise to be a promising intervention for managing the adverse effects of cancer and its treatments, with benefits including improved physical function, cardiorespiratory fitness, cancer-related fatigue, psychosocial well-being, and body composition [5-7]. Further, exercise has been associated with an increased tolerance for cancer medication, reduced risk of cancer recurrence, reduced all-cause mortality, as well as reduced breast, colon, and prostate cancer-specific mortalities [8-10]. Clear and consistent evidence aided in the development of survivor-specific exercise guidelines, the earliest of which recommended at least $150 \mathrm{~min}$ of moderate-intensity or $75 \mathrm{~min}$ of vigorous-intensity of aerobic exercise per week along with structured resistance training $[6,11,12]$. However, these guidelines have since been updated to be individualized, prescriptive, and less concerned with arbitrary targets [11,13-15]. Under current guidelines, each individual cancer survivor is recommended to be screened and assessed for sequelae, comorbidities, prior activity levels, and personal goals in order to inform the selection of the appropriate exercise dose, frequency, and modality [6, 11, 13-17].

Despite the abundance of evidence and detailed guidelines, most cancer survivors are not meeting exercise guideline recommendations $[4,12,18-22]$. Preliminary explorations into this phenomena have determined that oncology health care professionals (HCPs) often have limited awareness of exercise guidelines and report feeling underqualified to provide exercise guidance to survivors [13, 18, 22]. However, oncology HCPs have also expressed understanding exercise as an important component of survivorship care, as well as interest in receiving further education and multidisciplinary team support to address this cancer survivorship need
$[13,18]$. To date, preliminary investigations into facilitators and barriers of exercise for cancer survivors have been conducted in cohorts from countries including the USA, Australia, Norway, Korea, Canada, and the UK [20, 22-25] that are predominantly western civilizations with the exception of Korea. Survivor-specific barriers to exercise identified among these cohorts have included lack of time, fatigue, treatmentrelated side effects, and lack of education regarding exercise recommendations and benefits [13, 18, 22]. Key facilitators have included the ability to regain an aspect of control over one's health and mental wellbeing [22]. However, culture has a profound influence on health behaviors and existing findings may have limited generalizability to cancer survivors in Singapore.

Cancer survivorship care in Singapore is in its early stages [26]. Historically, Singapore's approach to cancer survivorship has been surveillance-focused and oncologist-centric, in contrast to the shared-care models of survivorship care often seen in North American and European countries [26, 27]. With a rising prevalence of survivors and recognition of structural changes needed to meet growing national survivorship needs, Singapore held its first cancer supportive and survivorship care forum in December of 2016. Several key principles for the nationwide improvement of cancer survivorship care were identified including the necessity of a survivor-centered focus, integrated and coordinated care, and a strong research infrastructure for the development of evidence-based programs [26]. In accordance with forum findings, the National Cancer Center, Singapore (NCCS) began the development of a new center dedicated to meeting the needs of Singapore's constantly growing cancer survivor population. A key area of interest for the new center is the implementation of evidencebased interventions to manage poorly understood survivorship issues, including standardized and structured exercise programs. However, there is a dearth of research regarding survivor exercise engagement and factors influencing engagement in Singapore that would be critical to the development and implementation of an effective exercise program for this unique population. Therefore, this study was broadly designed to (1) investigate barriers and facilitators to exercise among Singaporean cancer survivors who are undergoing, or have undergone, chemotherapy at the NCCS and to (2) assess Singaporean cancer survivors' exercise behaviors across the survivorship continuum.

\section{Methods}

\section{Study design}

This cross-sectional study was conducted at the NCCS between August and October 2019. The NCCS is the largest 
ambulatory cancer center in Singapore, treating up to $70 \%$ of all adult cancer patients. Ethics approval was granted by SingHealth Centralised Institutional Review Board (CIRB Ref: 2019/2528) prior to study commencement.

\section{Inclusion and exclusion criteria}

This study recruited cancer survivors who were at least 21 years old, able to read and understand English, diagnosed with any cancer of any stage (I-IV) by an oncologist, and who had received chemotherapy at the NCCS within the past 12 months prior to study enrollment. Cancer survivors were excluded if they had cognitive or severe psychiatric disorders that investigators judged to likely impair their ability to provide informed consent or answer questionnaires.

\section{Study procedures}

Survivors at the NCCS were identified by their oncologist, approached during either a routine chemotherapy session or consultation visit and screened for eligibility. Survivors who consented to participate were given a set of self-administered survey questionnaires in English which were collected upon completion. Each set of questionnaires took approximately 20-30 min to complete.

\section{Measures}

\section{Demographic and clinical information}

Demographic information (age, gender, height and weight, ethnicity, education, marital status, and employment status) and clinical information (cancer type, stage of cancer, Eastern Cooperative Oncology Group (ECOG) performance status, comorbidities, cancer treatments received, and time since chemotherapy completion) were collected through self-administered questionnaire.

\section{Exercise and physical activity behaviors}

Exercise and physical activity behavior were assessed through an investigator-designed recall questionnaire. Survivors were asked to classify weekly activity into two categories: exercise or physical activity. Exercise was defined as purposeful, prescriptive, programmed, and progressive activities of a specific nature [28]. Physical activity and exercise were further categorized into 3 levels of intensity: light (no noticeable change in breathing pattern), moderate (breath quickens but not out of breath, develop light sweat after approximately $10 \mathrm{~min}$, can talk but cannot sing), and vigorous (breathing is deep and rapid, develop sweat after several minutes, cannot say a few words without stopping to catch a breath) [11]. Survivors were asked to recall their exercise and physical activity behavior at each intensity level across four different cancer-related time periods: (1) prediagnosis, (2) post-diagnosis before chemotherapy, (3) during chemotherapy, and (4) post-chemotherapy. Post-chemotherapy activity was reported only by participants who had completed chemotherapy at the time of study participation. Information on activity type (walking, cycling, housework, etc.) and estimated weekly duration was collected in freeform text.

\section{Perceived exercise barriers and facilitators}

Cancer survivors' perceived barriers and facilitators to exercise were assessed using an investigator-designed questionnaire containing 20 barriers and 15 facilitators selected a priori based on findings from existing literature [20, 22-25, 29]. Participants were asked to select each facilitator and barrier they believed influenced their exercise behavior.

\section{Exercise guidance and education}

History of exercise education and guidance provision was assessed using 5 conditional, closed-ended questions (Fig. 1). Participants were asked whether they had previous exposure to information regarding exercise and cancer, were advised to exercise by a HCP (e.g., oncologist, physiotherapist, social worker) or fitness professional (e.g., personal trainer, instructor) following cancer diagnosis, and whether this information had been adequate to motivate them to initiate exercise. Finally, participant interest in a guided exercise program designed by study investigators for potential implementation at the new NCCS facility was assessed. This program was described as 3, 50-min sessions per week on a stationary bicycle: one supervised at a rehabilitation center and 2 unsupervised at home.

\section{Statistical analysis}

Statistical analyses were performed in SAS 9.3 (SAS Institute, Cary, NC). Descriptive statistics were used to describe demographic, clinical, and survey data. Categorical variables were presented as proportions and continuous variables were summarized as mean, standard deviation (SD), and range. For the purposes of data analysis, participants were divided into two subgroups: (1) those who were undergoing chemotherapy at the time of participation and (2) those who had completed therapy treatment at the time of participation. Differences between subgroups were assessed using chi-square tests for categorical demographic and clinical variables. Fisher's exact tests were used when cell counts were below 5 . Independent $t$-tests were used to compare age 
Fig. 1 Survivor exercise guidance and education survey

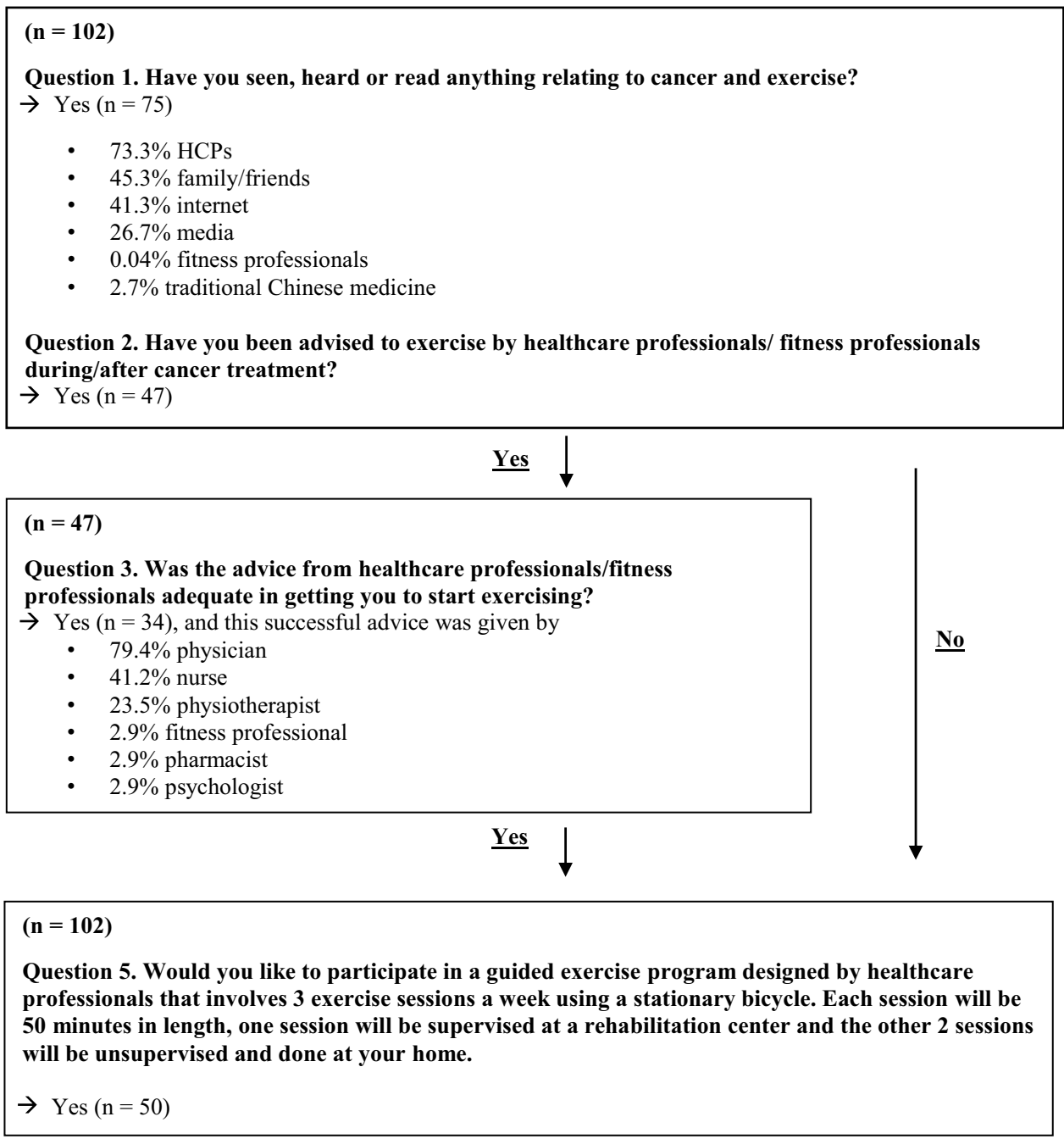

and body mass index (BMI) following confirmation of normality through QQ-plots. Differences between subgroups in the proportion of participants reporting each individual facilitator and barrier were compared using chi-square tests. Self-reported weekly duration of physical activity and exercise were summarized as median and interquartile range (IQR). Wilcoxon signed-rank test was used to test for differences in activity levels at each timepoint compared to activity levels prior to cancer diagnosis. Additionally, the proportion of respondents meeting NCCN cancer survivorship aerobic exercise guidelines at each time period was assessed. McNemar's test for paired samples was utilized to compare the proportion of participants meeting guidelines at each timepoint to the proportion meeting guidelines prior to cancer diagnosis. A $p$ value less than 0.05 was considered statistically significant.

\section{Results}

\section{Demographic and clinical characteristics}

A total of 221 survivors were identified and approached for participation in this study. Of the 221,202 were confirmed to meet eligibility criteria and $102(50.5 \%)$ consented to participate. Of the 102 study participants, 65 (63.7\%) were undergoing chemotherapy at the time participation and $37(36.3 \%)$ had completed chemotherapy. Participants were primarily Chinese $(84.3 \%)$, male $(52.9 \%)$, married $(72.5 \%)$, graduates/post-graduates $(38.2 \%)$, and not working at the time of participation $(43.1 \%)$ (Table 1$)$. The mean $( \pm$ SD) age of participants was $54.6 \pm 12.7$ and the mean $( \pm$ SD) BMI was $23.1 \pm 4.0$. Thirty-nine respondents $(38.2 \%)$ reported additional comorbidities, including hypertension (17.6\%), diabetes (15.7\%), and high cholesterol (13.7\%). No significant differences in demographic characteristics between participants undergoing chemotherapy at the time of participation and those who had completed chemotherapy were observed. 
Table 1 Demographic and clinical characteristics of study participants

\begin{tabular}{|c|c|c|c|c|}
\hline & $\begin{array}{l}\text { Undergoing chemotherapy } \\
(N=65)\end{array}$ & $\begin{array}{l}\text { Completed chemotherapy } \\
(N=37)\end{array}$ & $\begin{array}{l}\text { All survivors } \\
(N=102) \\
N(\%)\end{array}$ & $p$ value $^{\mathrm{a}}$ \\
\hline \multicolumn{5}{|l|}{ Demographic characteristics } \\
\hline Gender & & & & 0.512 \\
\hline Male & $36(55.4)$ & $18(48.6)$ & $54(52.9)$ & \\
\hline Female & $29(44.6)$ & $19(51.4)$ & $48(47.1)$ & \\
\hline Race & & & & 0.875 \\
\hline Chinese & $53(81.5)$ & $33(89.2)$ & $86(84.3)$ & \\
\hline Malay & $6(9.2)$ & $2(5.4)$ & $8(7.8)$ & \\
\hline Indian & $4(6.2)$ & $1(2.7)$ & $5(4.9)$ & \\
\hline Other & $2(3.1)$ & $1(2.7)$ & $3(2.9)$ & \\
\hline Marital status & & & & 0.926 \\
\hline Married & $48(73.8)$ & $26(70.3)$ & $74(72.5)$ & \\
\hline Single & $11(16.9)$ & $8(21.6)$ & 19 (18.6) & \\
\hline Divorced & $5(7.7)$ & $3(8.1)$ & $8(7.8)$ & \\
\hline Widowed & $1(1.5)$ & $0(0.0)$ & $1(1.0)$ & \\
\hline Living alone & & & & 0.250 \\
\hline No & $58(89.2)$ & $30(81.1)$ & $88(86.3)$ & \\
\hline Yes & $7(10.8)$ & $7(18.9)$ & $14(13.7)$ & \\
\hline Education level & & & & 0.699 \\
\hline Primary & $13(12.7)$ & $4(10.8)$ & $9(13.8)$ & \\
\hline Secondary & $30(29.4)$ & $10(27.0)$ & $20(30.8)$ & \\
\hline Pre-university & $20(19.6)$ & $6(16.2)$ & $14(21.5)$ & \\
\hline Graduate/post-graduate & $39(38.2)$ & $17(45.9)$ & $22(33.8)$ & \\
\hline Employment status & & & & 0.173 \\
\hline Not working & $44(43.1)$ & $14(37.8)$ & $44(43.1)$ & \\
\hline Full-time employment & $42(41.2)$ & $18(48.6)$ & $42(41.2)$ & \\
\hline Part-time employment & $9(8.8)$ & $1(2.7)$ & $9(8.8)$ & \\
\hline Self-employed & $7(6.9)$ & $4(10.8)$ & $7(6.9)$ & \\
\hline Age (years): mean \pm SD (range) & $54.0 \pm 12.6(21-86)$ & $55.8 \pm 12.9(31-84)$ & $54.6 \pm 12.7(21-86)$ & 0.493 \\
\hline \multicolumn{5}{|l|}{ Clinical characteristics } \\
\hline Cancer type & & & & $0.036^{*}$ \\
\hline Lower gastrointestinal tract & $19(29.2)$ & $7(18.9)$ & $26(25.5)$ & \\
\hline Breast & $15(23.1)$ & 7 (18.9) & $22(21.6)$ & \\
\hline Hematologic malignancies & $2(3.1)$ & $7(18.9)$ & $9(8.8)$ & \\
\hline Head and neck & $9(13.8)$ & $0(0.0)$ & $9(8.8)$ & \\
\hline Female reproductive organs & $4(6.2)$ & $4(10.8)$ & $8(7.8)$ & \\
\hline Hepatobiliary system & $5(7.7)$ & $3(8.1)$ & $8(7.8)$ & \\
\hline Upper gastrointestinal tract & $4(6.2)$ & $3(8.1)$ & $7(6.9)$ & \\
\hline Thorax & $4(6.2)$ & $2(5.4)$ & $6(5.9)$ & \\
\hline Genitourinary cancers & $1(1.5)$ & $3(8.1)$ & $4(3.9)$ & \\
\hline Soft tissue sarcoma & $2(3.1)$ & $1(2.7)$ & $3(2.9)$ & \\
\hline Cancer stage & & & & 0.059 \\
\hline I & $4(6.2)$ & $6(16.2)$ & $10(9.8)$ & \\
\hline II & $6(9.2)$ & $6(16.2)$ & $12(11.8)$ & \\
\hline III & $12(18.5)$ & $9(24.3)$ & $21(20.6)$ & \\
\hline IV & $43(66.2)$ & $15(40.5)$ & $58(56.9)$ & \\
\hline Not applicable ${ }^{\mathrm{b}}$ & $0(0.0)$ & $1(2.7)$ & $1(1.0)$ & \\
\hline
\end{tabular}


Table 1 (continued)

\begin{tabular}{|c|c|c|c|c|}
\hline & $\begin{array}{l}\text { Undergoing chemotherapy } \\
(N=65)\end{array}$ & $\begin{array}{l}\text { Completed chemotherapy } \\
(N=37)\end{array}$ & $\begin{array}{l}\text { All survivors } \\
(N=102) \\
N(\%)\end{array}$ & $p$ value $^{\mathrm{a}}$ \\
\hline ECOG performance status ${ }^{c}$ & & & & 0.857 \\
\hline 0 & $27(41.5)$ & $17(45.9)$ & $44(43.1)$ & \\
\hline 1 & $36(55.4)$ & $19(51.4)$ & $55(53.9)$ & \\
\hline 2 & $2(3.1)$ & $1(2.7)$ & $3(2.9)$ & \\
\hline \multicolumn{5}{|l|}{ Cancer treatments received } \\
\hline Chemotherapy & $65(100.0)$ & $37(100.0)$ & $102(100.0)$ & - \\
\hline Surgery & $35(53.8)$ & $27(73.0)$ & $62(60.8)$ & 0.057 \\
\hline Radiation & $15(23.1)$ & $8(21.6)$ & $23(22.5)$ & 0.866 \\
\hline Targeted & $9(13.8)$ & $8(21.6)$ & $17(16.7)$ & 0.311 \\
\hline Hormonal & $3(4.6)$ & $6(16.2)$ & $9(8.8)$ & 0.069 \\
\hline \multicolumn{5}{|l|}{ Comorbidities } \\
\hline Hypertension & $11(16.9)$ & $7(18.9)$ & $18(17.6)$ & 0.799 \\
\hline Diabetes & $11(16.9)$ & $5(13.5)$ & $16(15.7)$ & 0.649 \\
\hline High cholesterol & $8(12.3)$ & $6(16.2)$ & $14(13.7)$ & 0.581 \\
\hline Liver disease & $3(4.6)$ & $0(0.0)$ & $3(2.9)$ & 0.552 \\
\hline Osteoporosis & $2(3.1)$ & $2(5.4)$ & $4(3.9)$ & 0.620 \\
\hline Cardiovascular disease & $1(1.5)$ & $2(5.4)$ & $3(2.9)$ & 0.297 \\
\hline Glaucoma & $1(1.5)$ & $0(0.0)$ & $1(1.0)$ & 1.000 \\
\hline Lung disease & $1(1.5)$ & $0(0.0)$ & $1(1.0)$ & 1.000 \\
\hline Arthritis & $1(1.5)$ & $0(0.0)$ & $1(1.0)$ & 1.000 \\
\hline Kidney disease & $1(1.5)$ & $0(0.0)$ & $1(1.0)$ & 1.000 \\
\hline Others & $3(4.6)$ & $4(10.8)$ & $7(6.9)^{\mathrm{d}}$ & 0.665 \\
\hline BMI: mean \pm SD (range) & $23.0 \pm 4.4$ & $23.5 \pm 3.3$ & $23.1 \pm 4.0(13.1-35.7)$ & 0.484 \\
\hline $\begin{array}{l}\text { Time since chemotherapy comple- } \\
\text { tion }(N=37) \text { : mean } \pm \text { SD (range) }\end{array}$ & - & $4.6 \pm 3.9(0-12)$ & $4.6 \pm 3.9(0-12)$ & - \\
\hline
\end{tabular}

${ }^{a} \chi^{2}$ test was used to test for associations between chemotherapy completion and categorical variables, Fisher's exact tests were used when cell counts $<5$, and independent $t$-tests were used to test for associations between chemotherapy completion and the means of continuous variables

${ }^{\mathrm{b}}$ Cancer staging is unavailable for acute myeloid leukemia

${ }^{c}$ ECOG $0=$ fully active, able to carry on all pre-disease performance without restriction; ECOG $1=$ restricted in physically strenuous activity but ambulatory and able to carry out work of a light or sedentary nature, e.g., light housework and office work; ECOG $2=$ ambulatory and capable of all self-care but unable to carry out any work activities; up and about more than $50 \%$ of waking hours

${ }^{\mathrm{d}}$ Endometriosis $(n=1)$, dermatomyositis $(n=1)$, age-related macular degeneration on left eye $(n=1)$, myasthenia gravis $(n=1)$, thyroid $(n=1)$, psoriasis $(n=1)$, and PCOS $(n=1)$

${ }^{*} p$ value $<0.05$

Participants were primarily diagnosed with stage IV cancer $(60 \%)$. The most prevalent cancer types included lower gastrointestinal tract $(25.5 \%)$ and breast cancer (21.6\%). Participants undergoing chemotherapy at the time of study participation had significantly higher proportions of gastrointestinal tract and head and neck cancer, whereas participants who had completed chemotherapy had higher proportions of hematologic malignancies and genitourinary cancers. All participants received chemotherapy treatment, $60.8 \%$ had cancer-related surgery, and $22.5 \%$ received radiation therapy. Regarding disease impact, $43.1 \%$ of participants were fully active and able to continue pre-disease activity without restriction (ECOG score of 0), whereas $53.9 \%$ faced restrictions in physically strenuous activities but remained ambulatory (ECOG score of 1). Among the 37 participants who had completed chemotherapy at the time of study participation, the mean $( \pm \mathrm{SD})$ time since chemotherapy completion was $4.6 \pm 3.9$ months.

\section{Exercise behavior}

Prior to cancer diagnosis, $90.2 \%$ of all study participants reported physical activity and exercise satisfying NCCN cancer survivorship aerobic exercise guidelines (Table 2). 
This proportion was significantly reduced following cancer diagnosis $(69.6 \%, p<0.001)$ and remained reduced during chemotherapy $(65.7 \%, p<0.001)$. Among the 37 individuals who had completed chemotherapy, $75.7 \%$ met exercise guidelines after chemotherapy completion, which was not significantly different than the proportion in that subset who met guidelines prior to cancer diagnosis $(83.8 \%, p=0.180)$.

Moderate-intensity aerobic exercise was the most reported across all time periods. The median (IQR) weekly duration of moderate-intensity aerobic exercise across all participants decreased from 60 (0 to 157) $\mathrm{min} /$ week prior to cancer diagnosis to 0 ( 0 to 105 ) $\mathrm{min} /$ week following diagnosis $(p<0.001)$ and remained significantly lower during chemotherapy $(p<0.001)$ (Table 3$)$. However, among participants who had completed chemotherapy, the median weekly duration of moderate-intensity aerobic exercise after chemotherapy completion was not significantly different than prior to diagnosis $(p=0.297)$. The same pattern was observed for vigorous-intensity aerobic exercise, the median (IQR) weekly duration decreased from 0 (0 to 25$) \mathrm{min} / \mathrm{week}$ prior to cancer diagnosis to 0 ( 0 to 0$) \mathrm{min} /$ week following diagnosis $(p<0.001)$, and the median weekly duration after chemotherapy completion was not significantly different than prior to diagnosis $(p=0.539)$. Cancer diagnosis
Table 2 Proportion of respondents meeting NCCN cancer survivorship aerobic exercise guidelines $^{\mathrm{a}}$ across 4 cancer-related time periods

\begin{tabular}{|c|c|c|c|c|c|c|}
\hline Time period & $\begin{array}{l}\text { All respondents } \\
(N=102) \\
N(\%)\end{array}$ & $p$ value $^{\mathrm{b}}$ & $\begin{array}{l}\text { Undergoing } \\
\text { chemotherapy } \\
(n=65)\end{array}$ & $p$ value $^{\mathrm{b}}$ & $\begin{array}{l}\text { Completed } \\
\text { chemotherapy } \\
(N=37) \\
N\left(\%^{\mathrm{b}}\right)\end{array}$ & $p$ value $^{\mathrm{b}}$ \\
\hline Pre-diagnosis & $92(90.2)$ & Ref & $61(93.9)$ & Ref & $31(83.8)$ & Ref \\
\hline $\begin{array}{l}\text { Post-diagnosis, before } \\
\text { chemotherapy }\end{array}$ & $71(69.6)$ & $<0.001$ & $49(75.4)$ & 0.001 & $22(59.5)$ & 0.003 \\
\hline During chemotherapy & $67(65.7)$ & $<0.001$ & $46(70.8)$ & $<0.001$ & $21(56.8)$ & 0.002 \\
\hline Post-chemotherapy ${ }^{\mathrm{c}}$ & - & - & - & - & $28(75.68)$ & 0.180 \\
\hline
\end{tabular}

${ }^{a}$ Meeting the aerobic activity guideline is defined as at least 150 min of moderate-intensity or 75 min of vigorous physical activity and/or exercise per week

${ }^{\mathrm{b}} \mathrm{McNemar}$ 's test for paired samples was utilized to compare proportion of participants meeting activity guidelines at each timepoint to proportion at pre-diagnosis

${ }^{c}$ Post-chemotherapy proportions only include respondents who have completed chemotherapy $(N=37)$

Table 3 Median (IQR) reported weekly physical activity and exercise durations of participants by intensity level, across 4 cancer-related time periods $(N=102)$

\begin{tabular}{|c|c|c|c|c|c|c|c|c|}
\hline \multirow[t]{2}{*}{ Intensity } & \multicolumn{2}{|l|}{ Pre-diagnosis } & \multicolumn{2}{|c|}{$\begin{array}{l}\text { Post-diagnosis, before chemo- } \\
\text { therapy }\end{array}$} & \multicolumn{2}{|c|}{ During chemotherapy } & \multicolumn{2}{|c|}{ Post-chemotherapy $(N=37)^{\mathrm{b}}$} \\
\hline & $\begin{array}{l}\text { Duration-min/ } \\
\text { week, median } \\
\text { (IQR) }\end{array}$ & $p$ value $^{\mathrm{a}}$ & $\begin{array}{l}\text { Duration-min/ } \\
\text { week, median } \\
\text { (IQR) }\end{array}$ & $p$ value $^{\mathrm{a}}$ & $\begin{array}{l}\text { Duration-min/ } \\
\text { week, median } \\
\text { (IQR) }\end{array}$ & $p$ value $^{\mathrm{a}}$ & $\begin{array}{l}\text { Duration-min/ } \\
\text { week, median } \\
\text { (IQR) }\end{array}$ & $p$ value $^{\mathrm{a}}$ \\
\hline \multicolumn{9}{|l|}{ Exercise } \\
\hline Light & $(0.0-0.0)^{0.0}$ & Ref & $(0.0-0.0)^{0.0}$ & 0.502 & $(0.0-20.0)^{0}$ & 0.947 & $\begin{array}{r}0.0 \\
(0.0-20.0)\end{array}$ & 0.275 \\
\hline Moderate & $\begin{array}{r}60.0 \\
(0.0-157.5)\end{array}$ & Ref & $\begin{array}{r}0.0 \\
(0.0-105.0)\end{array}$ & $<0.001^{*}$ & $\begin{array}{r}0.0 \\
(0.0-140.0)\end{array}$ & $0.010^{*}$ & $\begin{array}{c}120.0 \\
(25.0-187.5)\end{array}$ & 0.297 \\
\hline Vigorous & $(0.0-25.0)^{0.0}$ & Ref & $(0.0-0.0)^{0.0}$ & $<0.001^{*}$ & $(0.0-0.0)^{0.0}$ & $<0.001 *$ & $(0.0-0.0)^{0.0}$ & 0.539 \\
\hline \multicolumn{9}{|c|}{ Physical activity } \\
\hline Light & $(0.0-50.0)^{0.0}$ & Ref & $\begin{array}{r}0.0 \\
(0.0-105.0)\end{array}$ & 0.445 & $\begin{array}{r}0.0 \\
(0.0-87.5)\end{array}$ & 0.321 & $\begin{array}{r}0.0 \\
(0.0-100.0)\end{array}$ & 0.953 \\
\hline Moderate & $\begin{array}{c}420.0 \\
(140.0-840.0)\end{array}$ & Ref & $\begin{array}{c}221.3 \\
(52.5-570.0)\end{array}$ & $<0.001 *$ & $\begin{array}{c}180.0 \\
(30.0-540.0)\end{array}$ & $0.010^{*}$ & $\begin{array}{c}220.0 \\
(20.0-455.0)\end{array}$ & $0.017 *$ \\
\hline Vigorous & $(0.0-0.0))^{0.0}$ & Ref & $(0.0-0.0)^{0.0}$ & 1.000 & $(0.0-0.0)^{0.0}$ & 1.000 & $(0.0-0.0)^{0.0}$ & 1.000 \\
\hline
\end{tabular}

${ }^{a} p$ values based on Wilcoxon signed rank test with pre-diagnosis value for each intensity level as the reference group

${ }^{\mathrm{b}}$ Post-chemotherapy data is only available for participants who completed chemotherapy at the time of study participation $(N=37)$

${ }^{*} p$ value $<0.05$ 
was also associated with statistically significant decreases in moderate-intensity physical activity; the median (IQR) decreased from $420(140.0-840.0)$ to $221(52.5-570.0)$ $\mathrm{min} /$ week $(p<0.001)$. This decrease was sustained during chemotherapy $(p=0.010)$ and, among the subset who had completed chemotherapy, after chemotherapy completion as well $(p=0.017)$. Across all time periods, light and moderate physical activity were more commonly reported than light and moderate exercise. Walking was the most common exercise modality reported across the cancer-related time periods (Table 4).

\section{Perceived exercise barriers and facilitators}

The most commonly reported barriers to exercise include adverse effects from treatment $(52.0 \%)$, lack of self-discipline (32.4\%), weather (43.1\%), and other health issues $(27.5 \%)$ (Table 5). The most commonly reported facilitators to exercise include the desire to remain healthy and productive (84.3\%), to improve sleep and mental well-being $(72.5 \%)$, encouraged by family and friends $(52.0 \%)$, having exercised prior to treatment with a desire to maintain this activity (50\%), increase confidence (50\%), help cope better with cancer treatment and side effects of the treatment (48\%), and to improve independence and self-control (47.1\%) (Table 5). No significant differences in facilitators or barriers were observed between participants undergoing chemotherapy and those who had completed chemotherapy.

\section{Exercise guidance and education}

Most respondents reported receiving information about cancer and exercise $(73.5 \% ; n=75 / 102)$, primarily from HCPs (e.g., oncologists, physiotherapists, social workers) (73.3\%; $n=55 / 75)$ (Fig. 1). Of the 47 participants (46.1\%; $n=47 / 102$ ) who reported having been advised to perform exercise by healthcare or fitness professionals following cancer diagnosis, all reported they believed exercise would be beneficial to their health and wellbeing prior to receiving guidance. Thirty-four of those participants $(72.3 \%$; $n=34 / 47$ ) reported the advice received had been adequate in getting them to start exercising. Fifty participants (49\%; $n=50 / 102$ ) expressed interest in the proposed investigatordesigned exercise program.

\section{Discussion}

Physical activity and exercise behavior of cancer survivors at NCCS were examined across the continuum of survivorship, and perceived barriers and facilitators to exercise (i.e., purposeful, prescriptive, programmed, and progressive activities targeting various bodily systems [28]) were described. Surprisingly, a large proportion of survivors in this cohort met the aerobic exercise NCCN guidelines prior to cancer diagnosis. This finding is distinct from observations among previous survivor populations in the USA, Canada, Germany, and Korea [4, 19, 21, 30-32], and is reflective of the generally healthy BMIs reported in this cohort, as well as
Table 4 Moderate and vigorous exercise modalities reported by study participants as free-text across 3 cancer-related time periods $(N=102)$

\begin{tabular}{|c|c|c|c|c|c|}
\hline \multicolumn{2}{|l|}{ Before diagnosis } & \multicolumn{2}{|c|}{ After diagnosis, before treatment } & \multicolumn{2}{|l|}{ During chemotherapy } \\
\hline Activity type & $N(\%)$ & Activity type & $N(\%)$ & Activity type & $N(\%)$ \\
\hline \multicolumn{6}{|c|}{ Moderate exercise types reported } \\
\hline Walk & $37(36.3)$ & Walk & $29(28.4)$ & Walk & $35(34.3)$ \\
\hline Jog/run & $15(14.7)$ & Jog/run & $4(3.9)$ & Jog/run & $2(2.0)$ \\
\hline Cycle & $6(5.9)$ & Cycle & $3(2.9)$ & Cycle & $2(2.0)$ \\
\hline Gym/physical training & $6(5.9)$ & Gym/physical training & $2(2.0)$ & Gym/physical training & $3(2.9)$ \\
\hline Sports & $4(3.9)$ & Sports & $1(1.0)$ & Sports & $1(1.0)$ \\
\hline Aerobics (unspecified) & $3(2.9)$ & Aerobics (unspecified) & $2(2.0)$ & Aerobics (unspecified) & $4(3.9)$ \\
\hline Swim & $3(2.9)$ & Swim & $0(0.0)$ & Swim & $0(0.0)$ \\
\hline Yoga & $2(2.0)$ & Yoga & $0(0.0)$ & Yoga & $0(0.0)$ \\
\hline Other $^{\mathrm{a}}$ & $4(3.9)$ & Other & $2(2.0)$ & Other & $0(0.0)$ \\
\hline \multicolumn{6}{|c|}{ Vigorous exercise types reported } \\
\hline Gym/physical training & $11(10.8)$ & Gym/physical Training & $3(2.9)$ & Gym/physical training & $0(0.0)$ \\
\hline Jog/run & $7(6.9)$ & Jog/run & $1(1.0)$ & Jog/run & $1(1.0)$ \\
\hline Swim & $9(8.8)$ & Swim & $1(1.0)$ & Swim & $1(1.0)$ \\
\hline Other $^{b}$ & $6(5.9)$ & Other & $3(2.9)$ & Other & $2(2.0)$ \\
\hline
\end{tabular}

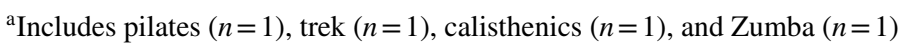

${ }^{\mathrm{b}}$ Includes basketball $(n=1)$, boxing $(n=1)$, calisthenics $(n=1)$, taekwondo $(n=1)$, trek $(n=1)$, and mountain biking $(n=1)$
} 
Table 5 Perceived facilitators and barriers to exercise among study participants

\begin{tabular}{|c|c|c|c|c|}
\hline Facilitators & $\begin{array}{l}\text { All survivors } \\
(N=102) \\
N(\%)\end{array}$ & $\begin{array}{l}\text { Undergoing chemotherapy } \\
(n=65) \\
N(\%)\end{array}$ & $\begin{array}{l}\text { Completed chemotherapy } \\
(n=37) \\
N(\%)\end{array}$ & $p$ value $^{\mathrm{a}}$ \\
\hline \multicolumn{5}{|l|}{ Personal } \\
\hline Remain healthy and productive & $86(84.3)$ & $57(87.7)$ & $29(78.4)$ & 0.261 \\
\hline Improve sleep and mental well-being & $74(72.5)$ & $50(76.9)$ & $24(64.9)$ & 0.249 \\
\hline Exercised prior to treatment with a desire to maintain this activity & $51(50.0)$ & $31(47.7)$ & $20(54.1)$ & 0.681 \\
\hline Increase confidence & $51(50.0)$ & $30(46.2)$ & $21(56.8)$ & 0.410 \\
\hline Cope better with cancer treatment and side effects from treatment & $49(48.0)$ & $33(50.8)$ & $16(43.2)$ & 0.539 \\
\hline Improve independence and self-control & $48(47.1)$ & $33(50.8)$ & $15(40.5)$ & 0.410 \\
\hline Reduce adverse effects from treatment & $45(44.1)$ & $29(44.6)$ & $16(43.2)$ & 1.000 \\
\hline Positive experience with exercise prior to treatment & $45(44.1)$ & $28(43.1)$ & $17(45.9)$ & 0.837 \\
\hline Prevent cancer recurrence & $40(39.2)$ & $22(33.8)$ & $18(48.6)$ & 0.205 \\
\hline Encouragement & $36(35.3)$ & $21(32.3)$ & $15(40.5)$ & 0.518 \\
\hline Sufficient time & $33(32.4)$ & $25(38.5)$ & $8(21.6)$ & 0.123 \\
\hline \multicolumn{5}{|l|}{ Social } \\
\hline $\begin{array}{l}\text { Interactions with other cancer patients through exercise } \\
\text { programs }\end{array}$ & $34(33.3)$ & $21(32.3)$ & $13(35.1)$ & 0.829 \\
\hline \multicolumn{5}{|l|}{ Environmental } \\
\hline Encouraged by family and friends to exercise & $53(52.0)$ & $32(49.2)$ & $21(56.8)$ & 0.539 \\
\hline Encouraged by healthcare professionals to exercise & $45(44.1)$ & $25(38.5)$ & $20(54.1)$ & 0.150 \\
\hline Accessible programs tailored to cancer patients & $37(36.3)$ & $23(35.4)$ & $14(37.8)$ & 0.833 \\
\hline Barriers & $\begin{array}{l}\text { All survivors } \\
(N=102) \\
N(\%)\end{array}$ & $\begin{array}{l}\text { Undergoing chemotherapy } \\
(N=65)\end{array}$ & $\begin{array}{l}\text { Completed chemotherapy } \\
(n=37)\end{array}$ & $p$ value $\mathrm{e}^{\mathrm{a}}$ \\
\hline \multicolumn{5}{|l|}{ Personal } \\
\hline $\begin{array}{l}\text { Adverse effects from treatment (e.g., lack of energy, fatigue, } \\
\text { numbness, tingling, muscle weakness, pain, depression, anxi- } \\
\text { ety, limited joint movement, vomiting) }\end{array}$ & $53(52.0)$ & $30(46.2)$ & $23(62.2)$ & 0.150 \\
\hline Lack of self-discipline & $33(32.4)$ & $19(29.2)$ & $14(37.8)$ & 0.387 \\
\hline Exercise limited by other health issues & $28(27.5)$ & $17(26.2)$ & $11(29.7)$ & 0.818 \\
\hline Fear of injury & $22(21.6)$ & $15(23.1)$ & $7(18.9)$ & 0.803 \\
\hline Lack of time & $21(20.6)$ & $17(26.2)$ & $4(10.8)$ & 0.078 \\
\hline Exercise is not a priority (e.g., work/family responsibilities) & $20(19.6)$ & $15(23.1)$ & $5(13.5)$ & 0.305 \\
\hline Lack of interest in exercise & $18(17.6)$ & $9(13.8)$ & $9(24.3)$ & 0.279 \\
\hline Exercise is not in routine & $17(16.7)$ & $12(18.5)$ & $5(13.5)$ & 0.591 \\
\hline Inconvenient exercise schedule & $11(10.8)$ & $9(13.8)$ & $2(5.4)$ & 0.320 \\
\hline $\begin{array}{l}\text { Uncertainty in use of fitness equipment and type of appropri- } \\
\text { ate exercises }\end{array}$ & $10(9.8)$ & $6(9.2)$ & $4(10.8)$ & 1.000 \\
\hline Unawareness of the need to exercise & $6(5.9)$ & $6(9.2)$ & $0(0.0)$ & 0.084 \\
\hline Exercise will make the cancer progress further & $2(2.0)$ & $2(3.1)$ & $0(0.0)$ & 0.533 \\
\hline \multicolumn{5}{|l|}{ Social } \\
\hline Lack of company & $23(22.5)$ & $12(18.5)$ & $11(29.7)$ & 0.222 \\
\hline \multicolumn{5}{|l|}{ Environmental } \\
\hline Weather (e.g., wet, warm, windy) & $44(43.1)$ & $27(41.5)$ & $17(45.9)$ & 0.683 \\
\hline Cost of exercising & $16(15.7)$ & $12(18.5)$ & $4(10.8)$ & 0.401 \\
\hline Lack of access to training facility or equipment & $11(10.8)$ & $6(9.2)$ & $5(13.5)$ & 0.522 \\
\hline Lack of appropriate exercise facility & $10(9.8)$ & $6(9.2)$ & $4(10.8)$ & 1.000 \\
\hline Lack of knowledgeable exercise staff & $10(9.8)$ & $6(9.2)$ & $4(10.8)$ & 1.000 \\
\hline Warned by healthcare professionals not to exercise & $7(6.9)$ & $5(7.7)$ & $2(5.4)$ & 1.000 \\
\hline Warned by family/friends not to exercise & $4(3.9)$ & $3(4.6)$ & $1(2.7)$ & 1.000 \\
\hline
\end{tabular}

${ }^{a} \chi^{2}$ test was used to test for associations between chemotherapy completion and barriers; Fisher's exact test was utilized when cell counts $<5$ 
the high levels of societal physical activity described in Singapore's National Health Survey (NHS). Limited existing investigations into patterns of physical activity in Singapore have shown travel-related activity to be a large contributor to physical activity, which could be related to Singapore's unique public transportation infrastructure and high taxes on private car ownership [33]. Cultural differences may also have influenced the comparatively high physical activity levels observed in this Singapore survivor cohort; however, further research is still greatly needed. In line with previous investigations [21, 30, 31, 34-36], the point of diagnosis remained a marker for the significant decline in both incidental physical activity and purposeful exercise. Therefore, even among this notably active cohort of survivors, opportunities for increased engagement in exercise throughout the survivorship continuum remain. Additionally, the point of diagnosis could serve as a key moment for the initiation of discussion regarding exercise.

As seen in previous investigations, the most commonly reported barrier to exercise was adverse effects from treatment, which encompassed a range of symptoms such as fatigue, muscle weakness, and pain (Table 5) [22, 37]. However, despite guidelines recommending the utilization of exercise to reduce cancer treatment-related sequelae [5-7], less than half of participants reported reductions in adverse events from treatment as a facilitator of exercise. These findings suggest a lack of education among Singaporean cancer survivors regarding the benefits of exercise that has been similarly observed in international investigations [22, 37]. This is further supported by the finding that less than half of participants reported having been directly advised to engage in exercise following their cancer diagnosis. Therefore, the effective implementation of an exercise intervention at the NCCS for cancer survivors will require evidence-based educational components to motivate and guide engagement. Optimistically, participant survey responses were generally reflective of positive perceptions of exercise and a receptiveness to exercise guidance. Less than one-fifth of participants reported a lack of interest in exercise and exercise not being a priority as barriers to exercise engagement, and the majority of participants reported facilitators such as the desire to remain healthy and productive and previous participation in exercise as facilitators. Additionally, contrary to previous investigations [22], only $20.6 \%$ of participants noted a lack of time as a barrier, suggesting a previously identified key barrier to engagement may be less prominent among this cohort. Further, 72\% (34/47) of participants who reported having been advised to exercise after cancer diagnosis reported that the advice was adequate in getting them to initiate exercise. Overall, these findings are supportive of the positive reception of exercise education and an appropriately designed and promoted exercise intervention for survivors at the NCCS.
In line with previous studies, walking was the most reported activity among participants [22-24, 38]. Although walking is an aerobic exercise activity, to maximize the benefits of exercise among cancer survivors and meet all components of the NCCN exercise guidelines, Singaporean cancer survivors must prioritize participation in resistance training activities. Resistance training, defined as muscle strengthening and muscle building exercises, is key for addressing important cancer-related side effects including bone and muscle loss, fragility, loss of physical function, and fall prevention, especially among metastatic cancer survivors who compose two-thirds of this study population [12, 13, 39-41]. Survivors with metastases have previously reported hesitancy to conduct exercise, particularly resistance training, without supervision due to issues including fear of fracture, bone metastases, and fragility [6, 7, 15, 42-44]. With $21.6 \%$ of cancer survivors reporting fear of injury as a barrier to exercise and an overwhelming majority engaging primarily in walking, individualized guidance and education regarding the appropriate and safe engagement in resistance training will be needed at the NCCS to optimize the benefits of exercise among survivors.

Although the necessity of survivor education regarding the benefits of exercise has been highlighted in this investigation, significant barriers to oncologist exercise promotion have been identified in literature that will require consideration during implementation at the NCCS. Oncologist lack of time and lack of knowledge regarding exercise and cancer survivorship [18] will likely be influential in Singapore where the oncologist-centric model of cancer care has historically placed the burden of addressing complex survivorship needs on the oncologists $[13,18,26]$. Therefore, not only is oncologist education regarding exercise guidelines for cancer survivors recommended, but multidisciplinary team support would be as well. Pilot studies examining the feasibility and acceptability of survivor-specific exercise intervention programs in other international cohorts have seen low referral and participation rates [40, 41, 45]. Filling the research-to-practice gap in Singapore exercise oncology and successfully integrating exercise into standard oncology care will further require the development of intentionally designed, standardized care pathways and implementation plans involving multidisciplinary team support [40, 41, 45].

The findings of this investigation should be interpreted with cautions. Physical activity and exercise are selfreported and subject to recall bias. Additionally, variability in the ability to recall based on the time since completion of each survivorship stage is likely reflected in these findings. The survey tool utilized to capture physical activity and exercise data was an investigator-developed, non-validated tool and thus not confirmed to be psychometrically sound. Additionally, given that resistance training is often poorly understood by cancer survivors and difficult to accurately 
capture through self-reported survey, only the formal assessment of aerobic exercise NCCN guideline adherence, and not resistance training guideline adherence, was conducted. However, all exercise types engaged were collected in freeform text as seen in Table 4. Further, the findings of this study did not control for the recency of treatments including surgery and radiation, which would likely influence reported exercise behavior. Additionally, given that only $50.5 \%$ of approached patients consented to participate in the study, the findings could be susceptible to sampling bias. Finally, participants were largely active prior to cancer diagnosis, viewed exercise in a positive light, were diagnosed with stage IV cancer, and therefore the findings should be interpreted within that context.

\section{Conclusion}

This is the first study investigating exercise activity, barriers, and facilitators among cancer survivors at NCCS, which is the largest ambulatory cancer center in Singapore. This study outlined necessity of increased exercise engagement following cancer diagnosis, the necessity of increased exercise education among survivors, outlined barriers and facilitators to exercise engagement, and revealed walking as the primarily exercise modality among survivors. The findings of this study provide vital preliminary data that can serve both in supporting and in informing the design of survivorspecific exercise interventions that can be integrated into Singapore's health care infrastructure at the NCCS in order to maximize the benefits of exercise among survivors.

Acknowledgements We thank Dr. Chiu Chin Ng and Ms. Jia Jue Foo for their assistance with this study.

Author contribution Conceptualization: Alexandre Chan, Patricia Neo, Mothi Babu Ramalingam, Benedict Tan, Ang Tee Lim, Kiley Loh; methodology: Alexandre Chan, Patricia Neo, Mothi Babu Ramalingam, Benedict Tan, Ang Tee Lim, Kiley Loh; formal analysis and investigation: Alexandre Chan, Kayleen Ports; writing—original draft: Alexandre Chan, Kayleen Ports, Raymond Javan Chan, Nicolas H. Hart; all authors commented on previous versions of the manuscript and all authors read and approved the final manuscript.

Funding Merck Sharp \& Dohme, International Neighbour of Choice (INOC) Grant (N-148-000-023-001).

Availability of data and material Data could be provided upon request.

Code availability Not applicable.

\section{Declarations}

Ethics approval This study was performed in accordance with the Declaration of Helsinki. Approval was granted by the Ethics Com- mittee SingHealth Centralised Institutional Review Board (CIRB Ref: 2019/2528).

Consent to participate Informed consent was obtained from all participants included in the study.

Consent for publication Patients signed informed consent regarding publishing their data.

Conflict of interest The authors declare no competing interests.

Open Access This article is licensed under a Creative Commons Attribution 4.0 International License, which permits use, sharing, adaptation, distribution and reproduction in any medium or format, as long as you give appropriate credit to the original author(s) and the source, provide a link to the Creative Commons licence, and indicate if changes were made. The images or other third party material in this article are included in the article's Creative Commons licence, unless indicated otherwise in a credit line to the material. If material is not included in the article's Creative Commons licence and your intended use is not permitted by statutory regulation or exceeds the permitted use, you will need to obtain permission directly from the copyright holder. To view a copy of this licence, visit http://creativecommons.org/licenses/by/4.0/.

\section{References}

1. Hashim D et al (2016) The global decrease in cancer mortality: trends and disparities. Ann Oncol 27(5):926-933

2. Harrington $C$ et al (2010) It's not over when it's over: long-term symptoms in cancer survivors-a systematic review. Int J Psychiatr Med 40(2):163-181

3. Siegel R, Miller K, Jemal A (2019) Cancer statistics. CA: Cancer J Clin 69(1):7-34

4. Blanchard CM et al (2008) Cancer survivors' adherence to lifestyle behavior recommendations and associations with healthrelated quality of life: results from the American Cancer Society's SCS-II. J Clin Oncol 26(13):2198-2204

5. Fuller JT et al (2018) Therapeutic effects of aerobic and resistance exercises for cancer survivors: a systematic review of metaanalyses of clinical trials. Br J Sports Med 52(20):1311

6. Hayes SC et al (2019) The Exercise and Sports Science Australia position statement: exercise medicine in cancer management. J Sci Med Sport 22(11):1175-1199

7. Dittus KL, Gramling RE, Ades PA (2017) Exercise interventions for individuals with advanced cancer: a systematic review. Prev Med 104:124-132

8. Ballard-Barbash R et al (2012) Physical activity, biomarkers, and disease outcomes in cancer survivors: a systematic review. J Natl Cancer Inst 104(11):815-840

9. Gunnell AS et al (2017) Physical activity and survival among long-term cancer survivor and non-cancer cohorts. Front Public Health 5:19

10. Bonn SE et al (2015) Physical activity and survival among men diagnosed with prostate cancer. Cancer Epidemiol Biomarkers Prev 24(1):57-64

11. National Comprehensive Cancer, N. and et al (2018) NCCN clinical practice guidelines in oncology: survivorship

12. Schmitz KH et al (2010) American College of Sports Medicine roundtable on exercise guidelines for cancer survivors. Med Sci Sports Exerc 42(7):1409-1426 
13. Stout NL, et al (2017) A systematic review of exercise systematic reviews in the cancer literature (2005-2017). PM R 9(9S2):S347-S384

14. Pollán M et al (2020) Exercise and cancer: a position statement from the Spanish Society of Medical Oncology. Clin Transl Oncol 22(10):1710-1729

15. Campbell KL et al (2019) Exercise guidelines for cancer survivors: consensus statement from international multidisciplinary roundtable. Med Sci Sports Exerc 51(11):2375-2390

16. Runowicz CD et al (2016) American Cancer Society/American Society of Clinical Oncology breast cancer survivorship care guideline. J Clin Oncol 34(6):611-635

17. Skolarus TA et al (2014) American Cancer Society prostate cancer survivorship care guidelines. CA Cancer J Clin 64(4):225-249

18. Nadler M et al (2017) Oncology care provider perspectives on exercise promotion in people with cancer: an examination of knowledge, practices, barriers, and facilitators. Support Care Cancer 25(7):2297-2304

19. Tarasenko Y, Chen C, Schoenberg N (2017) Self-reported physical activity levels of older cancer survivors: results from the 2014 National Health Interview Survey. J Am Geriatr Soc 65(2):e39-e44

20. Kang DW et al (2014) Exercise barriers in Korean colorectal cancer patients. Asian Pac J Cancer Prev 15(18):7539-7545

21. Chung JY et al (2013) Patterns of physical activity participation across the cancer trajectory in colorectal cancer survivors. Support Care Cancer 21(6): 1605-1612

22. Clifford BK et al (2018) Barriers and facilitators of exercise experienced by cancer survivors: a mixed methods systematic review. Support Care Cancer 26(3):685-700

23. Gjerset GM et al (2011) Interest and preferences for exercise counselling and programming among Norwegian cancer survivors. Eur J Cancer Care (Engl) 20(1):96-105

24. Karvinen KH et al (2007) Exercise programming and counseling preferences in bladder cancer survivors: a population-based study. J Cancer Surviv 1(1):27-34

25. Smith L, et al (2017) Cancer survivors' attitudes towards and knowledge of physical activity, sources of information, and barriers and facilitators of engagement: a qualitative study. Eur $\mathbf{J}$ Cancer Care 26(4):e12641

26. Loh KW et al (2018) Cancer supportive and survivorship care in Singapore: current challenges and future outlook. J Glob Oncol $4: 1-8$

27. McCabe MS et al (2013) American Society of Clinical Oncology statement: achieving high-quality cancer survivorship care. J Clin Oncol 31(5):631-640

28. Hart NH, Galvão DA, Newton RU (2017) Exercise medicine for advanced prostate cancer. Curr Opin Support Palliat Care 11(3):247-257

29. Gho SA et al (2014) Exercise bra discomfort is associated with insufficient exercise levels among Australian women treated for breast cancer. Support Care Cancer 22(3):721-729

30. Eng L et al (2018) Patterns, perceptions, and perceived barriers to physical activity in adult cancer survivors. Support Care Cancer 26(11):3755-3763
31. Schmidt ME et al (2017) Self-reported physical activity behavior of breast cancer survivors during and after adjuvant therapy: 12 months follow-up of two randomized exercise intervention trials. Acta Oncol 56(4):618-627

32. Steindorf $\mathrm{K}$ et al (2020) Change patterns and determinants of physical activity differ between breast, prostate, and colorectal cancer patients. Support Care Cancer 28(7):3207-3218

33. Win AM et al (2015) Patterns of physical activity and sedentary behavior in a representative sample of a multi-ethnic South-East Asian population: a cross-sectional study. BMC Public Health $15: 318$

34. Bock C et al (2013) Walking, bicycling, and sports in postmenopausal breast cancer survivors-results from a German patient cohort study. Psychooncology 22(6):1291-1298

35. Fassier $P$ et al (2016) Variations of physical activity and sedentary behavior between before and after cancer diagnosis: results from the prospective population-based NutriNet-Santé cohort. Medicine (Baltimore) 95(40):e4629

36. Sheill $\mathrm{G}$ et al (2018) The views of patients with metastatic prostate cancer towards physical activity: a qualitative exploration. Support Care Cancer 26(6):1747-1754

37. Avancini A et al (2020) Factors influencing physical activity in cancer patients during oncological treatments: a qualitative study. Integr Cancer Ther 19:1534735420971365

38. Avancini A, et al (2020) Exercise levels and preferences in cancer patients: a cross-sectional study. Int J Environ Res Public Health 17(15)

39. Santa Mina D et al (2019) Development, implementation, and effects of a cancer center's exercise-oncology program. Cancer 125(19):3437-3447

40. Kennedy MBS, Galvão D, Singh F, Spry N, Davis M, Chee R et al (2020) If you build it, will they come? Evaluation of a co-located exercise clinic and cancer treatment centre using the RE-AIM framework. Eur J Cancer Care 29(4):e13251

41. Kennedy MA et al (2020) We have the program, what now? Development of an implementation plan to bridge the researchpractice gap prevalent in exercise oncology. Int J Behav Nutr Phys Act 17(1):128

42. Galvão DA et al (2018) Exercise preserves physical function in prostate cancer patients with bone metastases. Med Sci Sports Exerc 50(3):393-399

43. Beijer S et al (2008) Determinants of overall quality of life in preterminal cancer patients. Int J Cancer 123(1):232-235

44. Weller S, et al (2021) Exercise for individuals with bone metastases: a systematic review. Crit Rev Oncol Hematol 103433

45. Casa DJ, Clarkson PM, Roberts WO (2005) American College of Sports Medicine roundtable on hydration and physical activity: consensus statements. Curr Sports Med Rep 4(3):115-127

Publisher's Note Springer Nature remains neutral with regard to jurisdictional claims in published maps and institutional affiliations. 